genetic and physiological information to aid conservation and to recover the species in the wild.

QIAO YAOMEI and LIU JiAN Key Laboratory for Plant Diversity and Biogeography of East Asia, Kunming Institute of Botany, Chinese Academy of Sciences, Kunming, Yunnan, China

Gong Xun (D) No. 132, Lanhei Road, Panlong District, Kunming City, Yunnan Province, China

E-mailgongxun@mail.kib.ac.cn

\section{Capacity Building for Conservation 2019}

One of the biggest challenges for conservation in the 21st century is establishing and maintaining sufficient capacity to tackle the growing number of environmental pressures around the world. This capacity needs to provide individuals, communities and organizations with the skills, knowledge and information for undertaking the wide range of tasks required for conserving biodiversity. To build this capacity we need to develop, disseminate and evaluate a diversity of relevant and cost-effective methods. This can only be achieved by ensuring we learn from initiatives in different regions and have the opportunities to communicate with others building capacity for conservation. Three regional conferences-in Colombia in 2013, Kenya in 2015 and India in 2017-have facilitated the exchange of ideas on this subject. From these meetings a number of cross-cutting issues emerged: (1) A range of new and regionally relevant capacity building tools is required to scale-up efforts to build capacity for conservation, including the further development of technological solutions such as e-learning and tools that are accessible in a range of languages. (2) Capacity building strategies and initiatives must take into account the often different enabling environments in different regions. (3) There is a need for the development of methods to evaluate capacity building and identify best practice. (4) There is an urgent need for conversations between donors and grantees to gauge expectations and guide the appropriate focus of funding streams. To directly tackle these issues, a global conference will take place at the Zoological Society of London on 30 July-1 August 2019. See https://conservationcapacity2019.co.uk/ for further information and the preliminary programme. The presentations will be live-streamed and made available on the conference website, and a series of short practical guides providing a comprehensive review of all major capacity building methods and associated research evidence will be published. For further information or to discuss attendance please contact Mark O'Connell (moconnell@glos.ac.uk).

MARK O'CONNELL University of Gloucestershire, Cheltenham, Gloucestershire, UK. E-mail moconnell@glos.ac.uk

JAKE BURTON Fauna \& Flora International, Cambridge, UK

\section{Discovery of new populations of the Vulnerable plant Neopicrorhiza scrophulariiflora in Yunnan, China}

Neopicrorhiza scrophulariiflora (Pennell) D. Y. Hong, a perennial herb of the monotypic genus Neopicrorhiza in the family Scrophulariaceae, occurs in Bhutan, Nepal, northeast India, and south-west China. It was first described as Picrorhiza scrophularifflora by Francis Whittier Pennell in 1943 (Academy of Natural Sciences of Philadelphia Monographs, 5, 65). The species was recognized as a class II protected species in the National Key Protected Wild Plants List of China in 1999 and categorized as Vulnerable on the China Species Red List in 2004. In 2010 the species was identified as one of 62 plant species with extremely small populations in Yunnan, China. In 2001, based on the field knowledge of experts, $N$. scrophularifflora was evaluated to occur in $<5$ localities in China, with an estimated population of $<1$,ooo mature individuals.

To obtain up-to-date information on N. scrophulariiflora in China a total of six field surveys were carried out in the southwest during 2016-2018, with the joint support of the National Natural Science Foundation of China (Grant No. 31570212, 31770228), the National Key Programme of the Ministry of Science and Technology (Grant No. 2017FY100100), and the Talent Project of Yunnan (Grant No. 2015HBo92). Two flowering populations of $N$. scrophulariiflora were discovered in Hengduan Mountains in western Yunnan, growing on gravelly grassland at an altitude of $4,160 \mathrm{~m}$. One population, in Gongshan County, has 469 individuals and another population, in Deqin County, has 437 individuals. The discovery of these new populations is promising news for the conservation of the species. However, our survey and information obtained from interviews with local people indicated that the main threats to these populations are anthropogenic use (the roots are used in traditional medicine) and road construction, and hence habitat loss. Effective measures are required to protect these new populations.

The Kunming Institute of Botany is now carrying out further studies on the population dynamics and genetic diversity of $N$. scrophularifflora across the two populations, to improve management and protection of this species in China. In collaboration with staff of nature reserves, we are also planning to collect seeds of $N$. scrophulariiflora for propagation and future restoration of wild populations. Using species distribution models we plan to identify and explore other sites in China where the species could potentially grow.

RoNG LI Key Laboratory for Plant Diversity and Biogeography of East Asia, Kunming Institute of Botany, Chinese Academy of Sciences, Kunming, China.E-mail lirong@mail.kib.ac.cn

YUN XIONG and JUN SUN Gongshan Branch of Gaoligongshan National Nature Reserve, Gongshan, China 